EPJ Web of Conferences 70, 00057 (2014)

DOI: $10.1051 /$ epjconf/ 20147000057

C Owned by the authors, published by EDP Sciences, 2014

\title{
Open heavy flavor and quarkonia measurements in heavy-ion collisions at RHIC
}

\author{
Jaroslav Bielcik ${ }^{1, a}$ \\ ${ }^{1}$ Faculty of Nuclear Sciences and Physical Engineering, Czech Technical University in Prague, Brehova 7, \\ Prague, 11519, Czech Republic
}

\begin{abstract}
The properties of the hot and dense nuclear matter produced at RHIC in heavy-ion collisions can be investigated in multiple ways by heavy flavor production. The STAR and PHENIX experiments have excellent capability to study both open heavy flavor and quarkonia. Heavy quarks are produced in early stage of the collisions and the mechanisms of their interaction with nuclear matter are not yet well understood. The open heavy flavor hadrons can be studied using electrons from their semileptonic decays or via direct reconstruction through their hadronic decay channels. The heavy quarkonia production is expected to be sequentially suppressed depending on the temperature of the produced nuclear matter. However, cold nuclear matter effects play an important role and have to be well understood. In this paper we report recent results from the RHIC heavyion program on non-photonic electrons, direct reconstruction of charm mesons, $\mathrm{J} / \psi$ as well as $\Upsilon$ in $\mathrm{p}+\mathrm{p}, \mathrm{d}+\mathrm{Au}$ and $\mathrm{Au}+\mathrm{Au}$ collisions at $\sqrt{s_{N N}}=200 \mathrm{GeV}$.
\end{abstract}

\section{Introduction}

The Relativistic Heavy Ion Collider (RHIC) at Brookhaven National Laboratory in USA has been since the year 2000 systematically studying the properties of hot and dense nulear matter by the means of heavy-ion collisions. So far many various collision systems have been studied: polarized proton-proton collisions up to center of mass energy $\sqrt{s}=500 \mathrm{GeV}, \mathrm{Au}+\mathrm{Au}$ collisions at $\sqrt{s_{N N}}=7.7$ $200 \mathrm{GeV}, \mathrm{Cu}+\mathrm{Au}$ and $\mathrm{U}+\mathrm{U}$ at $\sqrt{s_{N N}}=200 \mathrm{GeV}$. The theory of strong interaction, Quantum Chromodynamics (QCD), predicts that nuclear matter undergoes at some critical temperature and nuclear density a phase transition to the state of quark-gluon plasma from hadronic matter. Several experimental phenomena are proposed to be a consequence of this phase transition, such as high- $p_{T}$ hadron suppression and back-to-back di-hadron suppression ("jet quenching") in central Au+Au collisions, large hadron elliptic flow $\left(v_{2}\right)$ and constituent quark number scaling of $v_{2}$. These observables are related to the production of lights quarks and gluons, that are dominating the particle production. At the initial phase of collision, in hard scatterings, it is also possible to produce the heavier quarks: charm and bottom. These quarks do not change their mass during the collision. Due to their large mass, their production is expected to be well described and calculable by perturbative QCD (pQCD). The measurements of heavy quark production in $\mathrm{Au}+\mathrm{Au}$ collisions allow to extract properties of the produced nuclear matter. Additionally, phenomena such as color Debye screeing of di-quark potential

a e-mail: jaroslav.bielcik@fjfi.cvut.cz

This is an Open Access article distributed under the terms of the Creative Commons Attribution License 2.0, which permits unrestricted use, distribution, and reproduction in any medium, provided the original work is properly cited. 


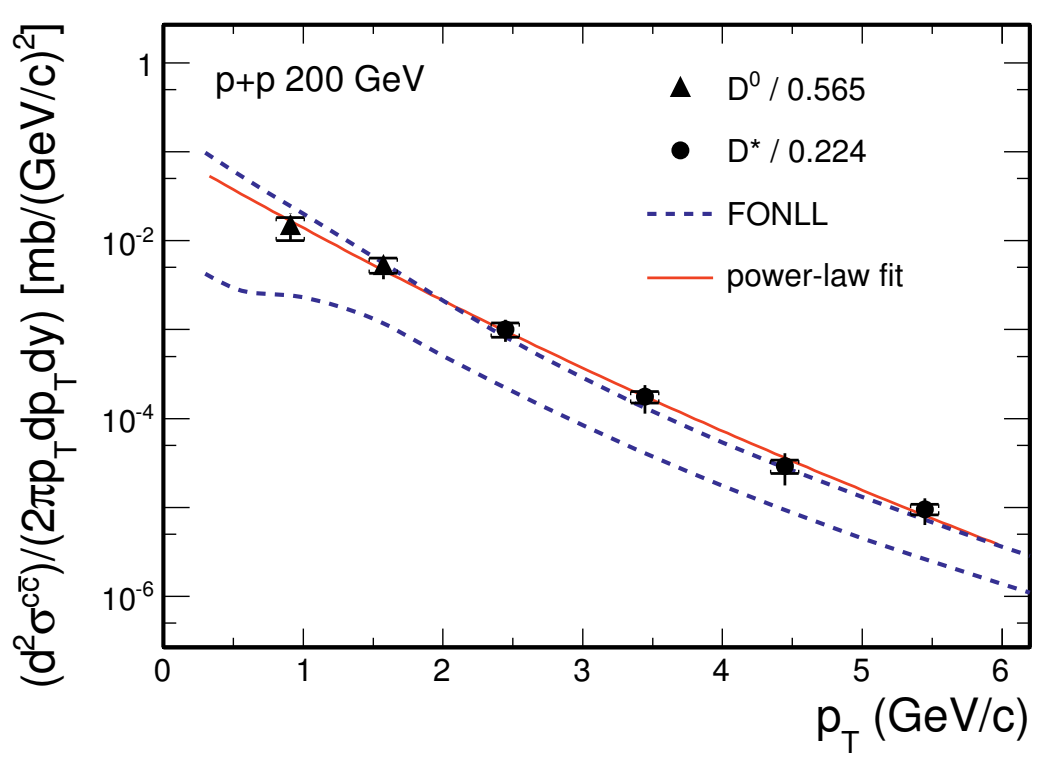

Figure 1. The invariant $c \bar{c}$ production cross section extracted from $D^{0}$ and $D^{*}$ yields in $\mathrm{p}+\mathrm{p}$ collisions at $\sqrt{s}=$ $200 \mathrm{GeV}$ in the STAR experiment compared with the FONLL calculations [6]. Taken from Ref. [4].

play a very important role in production of quarkonia and lead to directly observable effects in $\mathrm{J} / \psi$ and $\Upsilon$ production. However, the effects of the hot and dense nuclear matter have to be disentagled from effects that are already present in normal nuclear matter, the so called cold nuclear matter effects. Without a detailed experimental determination of these effects from $\mathrm{d}+\mathrm{Au}$ collisions it is not possible to interpret heavy-ion results.

In next sections the selection of the recent results on heavy flavor production in the STAR and PHENIX experiments is discussed.

\section{Open heavy flavor}

Open heavy flavor signals at RHIC can be measured in several ways: directly by the reconstruction of charm mesons and indirectly by measuring the electrons from semileptonic decays of charm and bottom mesons referred to as non-photonic electrons (NPE). So far the detector systems in the STAR and PHENIX experiments do not allow a topological reconstruction of hadronic decays of charm mesons, due to absence of a precise measurement of displaced vertices. Therefore such channels can be accessed only by statistical analysis and subtraction of combinatorial background. The STAR experiment has previously reported $D^{0}$ measurements in $\mathrm{d}+\mathrm{Au}, \mathrm{Cu}+\mathrm{Cu}$ and $\mathrm{Au}+\mathrm{Au}$ collisions at $200 \mathrm{GeV}$ [1-3]. Recently STAR has presented results from $D^{0}, D^{*}$ in $\mathrm{p}+\mathrm{p}$ collisions at $200 \mathrm{GeV}$ [4] and $500 \mathrm{GeV}$ [5] as well as the $D^{0}$ spectra and flow measuremets in Au+Au collisions at $200 \mathrm{GeV} \mathrm{[5].}$ The invariant $c \bar{c}$ production cross section as a function of transverse momentum $\left(p_{T}\right)$ extracted from $D^{0}$ and $D^{*}$ yields (using branching fractions) in $\mathrm{p}+\mathrm{p}$ collisions is presented in Fig. 1 . The data are compared to the FONLL calculations [6]. It is observed that FONLL calculations [6] describe the charm spectra well within the uncertainity bands of data and calculations. 


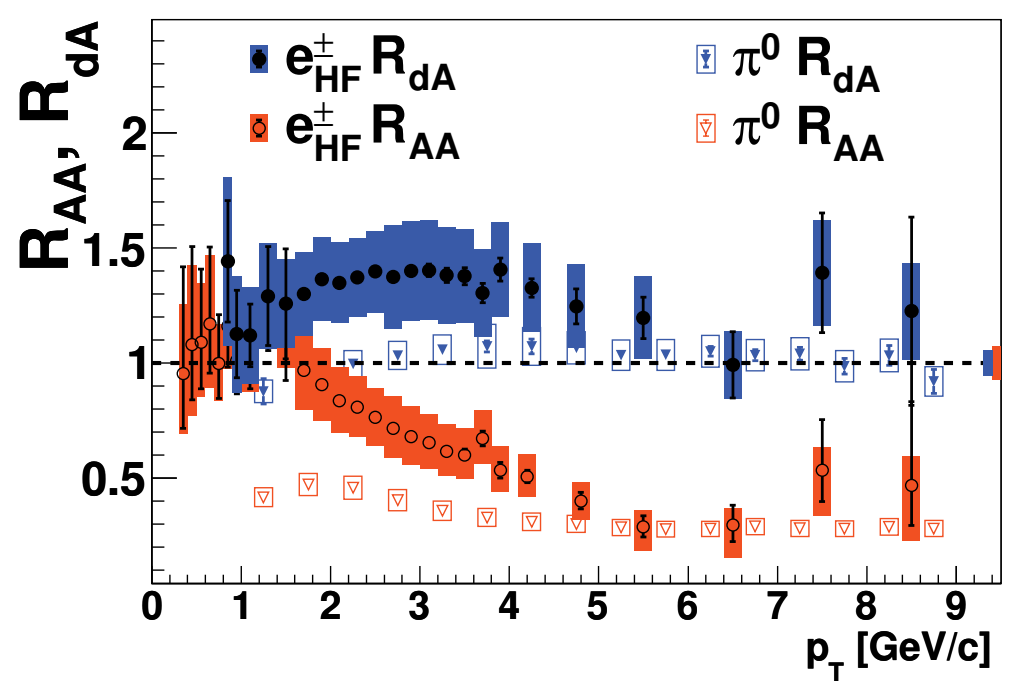

Figure 2. The nuclear modification factors $R_{d A}$ and $R_{A A}$ for minimum bias d+Au and Au+Au collisions measured by the PHENIX experiment, for the $\pi^{0}$ and non-photonic electrons. Taken from Ref. [12].

Non-photonic electrons were measured both by the STAR and PHENIX experiments. The most remarkable observation is the strong suppression of high- $p_{T}$ non-photonic electron production in central $\mathrm{Au}+\mathrm{Au}$ collisions [7, 8]. The strength of this suppression is similar to that of the light hadrons. This was unexpected and it contradicts to expectations of dead-cone effect that leads to smaller energy loss of heavy quarks when compared to light quarks and gluons. Since the non-photonic electron spectrum has contributions from both charm and as well bottom decays, it is important to disentangle the contributions from both channels experimentally. This was done in a model dependent way by means of non-photonic electron-hadron azimuthal correlations [9]. The relative contribution of electrons from bottom semileptonic decays in the total spectrum is about $50 \%$ at the transverse momentum of about $5 \mathrm{GeV} / c$ in $200 \mathrm{GeV} \mathrm{p}+\mathrm{p}$ collisions. This is consistent with the FONLL QCD calculations. The PHENIX experiment could also determine the flow of the non-photonic electrons. The non-zero value of the eliptic flow in the region $1-3 \mathrm{GeV} / c$ was observed. It is still a theoretical challenge to describe the nuclear modification factor and eliptic flow of the NPE in a consistent way together with the light flavor hadrons spectra. The role of the radiative and collisional energy loss of heavy quarks as well as effects of cold nuclear matter on their spectra are not well known.

The non-photonic electron transverse momentum spetra measured by STAR and PHENIX in $\mathrm{p}+\mathrm{p}$ collsions at $200 \mathrm{GeV}[8,10,11]$ are consistent with each other as well as with the upper bound of the FONLL calculation. Recently PHENIX reported a detailed study of cold nuclear matter effects in open heavy flavor production by measuring the NPE spectra in $\mathrm{d}+\mathrm{Au}$ collisions at $200 \mathrm{GeV}$ for various cetralities of collisions [12]. It was observed that cold nuclear matter modification factor $R_{d A u}$ is about 1.3-1.5 for $1<p_{T}<4 \mathrm{GeV} / c$ in more central collisions, see Fig. 2. This indicates possible cold nuclear matter enhancement. Such enhancement is not present in $\pi^{0}$ spectra. 


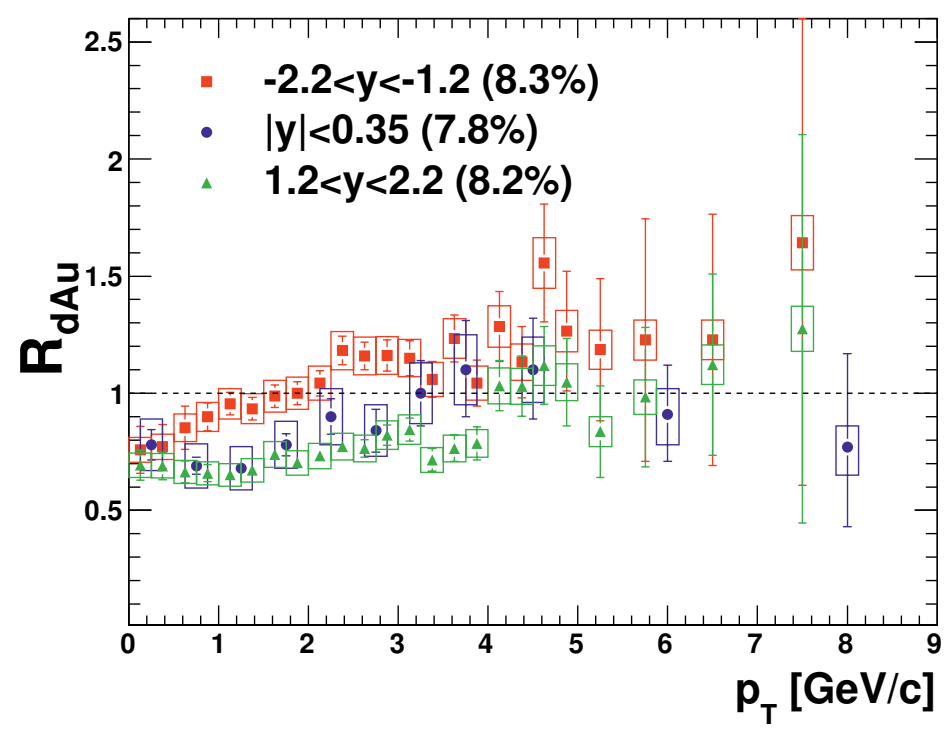

Figure 3. $\mathrm{J} / /$ psi nuclear modification factor $R_{d A u}$ as a function of $p_{T}$ for $0-100 \%$ centrality integrated d+Au collisions at forward, mid and backward rapidity measured by the PHENIX experiment. Taken from Ref. [17].

\section{$3 \mathrm{~J} / \psi$ measurements}

The $\mathrm{J} / \psi$ suppression in heavy-ion collisions is considered to be a sensitive probe of color deconfinement in QGP [13]. There are no unique predictions about the exact melting temperatures of various quarkonium states and several competing mechanisms having opposite effects on measured yields, could be important. These include cold nuclear matter effects (nuclear interaction break-up, shadowing) and recombination. Previous measurements at RHIC showed that the suppression of $\mathrm{J} / \psi$ as a function of centrality is similar to that observed at the CERN SPS energy [14]. This is puzzling since the temperature and energy density reached in these collisions is significantly lower than at RHIC [15]. PHENIX also observed that suppression is stronger at forward rapidity than at midrapidity. Therefore complex measurements are needed to interpret the suppression data properly.

The PHENIX experiment performed systematic effort to understand the cold nuclear matter effects of charmonium production. The $\mathrm{J} / \psi$ production in $\mathrm{d}+\mathrm{Au}$ collsions at $200 \mathrm{GeV}$ energy in central and forward and backward rapidity was measured $[16,17]$. It was observed that using the EPS09 nuclear parton distribution functions (nPDF) with $\sigma_{b r}=4 \mathrm{mb}$ can describe the central collisions well but not the peripheral ones. The nuclear modification factor in $\mathrm{d}+\mathrm{Au}$ collisions is similar for mid and forward rapidity, see Fig. 3. The suppression is observed for $p_{T}<4 \mathrm{GeV} / c$ and $R_{d A u} \approx 1$ for $p_{T}>4 \mathrm{GeV} / c$. For the backward rapidity is $R_{d A u}>1$ for $p_{T}>2 \mathrm{GeV} / c$.

The STAR experiment has measured the production of $\mathrm{J} / \psi$ at high $p_{T}[18,19]$. It is observed that although the suppression of charmonium is stronger for more central collisions, the $R_{A A} \mathrm{for} \mathrm{J} / \psi$ is seen to increase with increasing $p_{T}$. STAR has also measured azimuthal anisotropy of $\mathrm{J} / \psi$ up to $p_{T} \approx 8 \mathrm{GeV} / c$ [19]. The results are consistent with small or no flow of $\mathrm{J} / \psi$ at RHIC energies. 


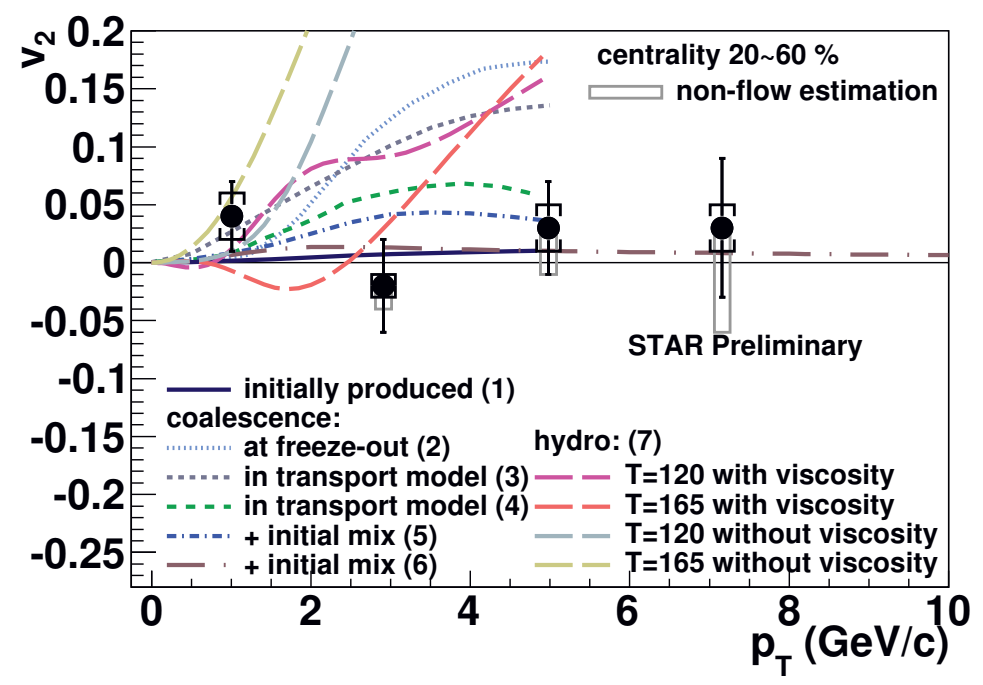

Figure 4. The transverse momentum dependence of azimuthal anisotropy parameter $v_{2}$ measured by the STAR experiment in 20-60\% most central Au+Au collisions. Taken from Ref. [19].

\section{$4 \Upsilon$ measurements}

The measurement of the $\Upsilon$ production in heavy-ion collisions is of a great interest, because it is expected that $\Upsilon(1 S)$ state does not dissociate at RHIC energies, but $\Upsilon(2 S, 3 S)$ do. This could provide a model independent thermometer of heavy-ion collisions. STAR reported measurements in $\mathrm{p}+\mathrm{p}$ [20], $\mathrm{d}+\mathrm{Au}$ [21] and $\mathrm{Au}+\mathrm{Au}$ [22] collisions. However, so far it was not possible to adress each $\Upsilon$ state separately. Recently STAR reported $\Upsilon$ results from Year $2010 \mathrm{Au}+\mathrm{Au} 200 \mathrm{GeV}$ collisions [19], see Fig.5. The increase of suppression as a function of the number of participants was observed. The $\Upsilon(1 \mathrm{~S}, 2 \mathrm{~S}, 3 \mathrm{~S})$ suppression was consistent with the scenario that $2 \mathrm{~S}, 3 \mathrm{~S}$ and excited states completely melt. Future measurements with recently installed Muon Telescope Detector in STAR will make possible to separate $\Upsilon$ states in di-muon channel at midrapidity.

\section{Summary}

In summary, STAR and PHENIX have measured heavy flavor production at RHIC in a complex way. The open heavy flavor production in $\mathrm{p}+\mathrm{p}$ collisions is well described by the FONLL calculations. The strong suppression of non-photonic electrons, similar to that of light hadrons, is observed as well as non-zero eliptic flow. The cold nuclear effects could play an important role at medium $p_{T}$ for non-photonic electron production.

The role of cold nuclear matter effects in charmonium production was studied in a detailed way by the PHENIX experiment. The suppression of $\mathrm{J} \psi$ is decreasing with larger $p_{T}$. The $\Upsilon$ suppression was observed in central $\mathrm{Au}+\mathrm{Au}$ collisions and it is consistent with only $1 \mathrm{~S}$ state survival. The new upgrades of the STAR and PHENIX detectors will allow to perform more precise heavy flavor measurements in the near future at RHIC.

This work was supported by the grant INGO LA09013 of the Ministry of Education, Youth and Sports of the Czech Republic. 


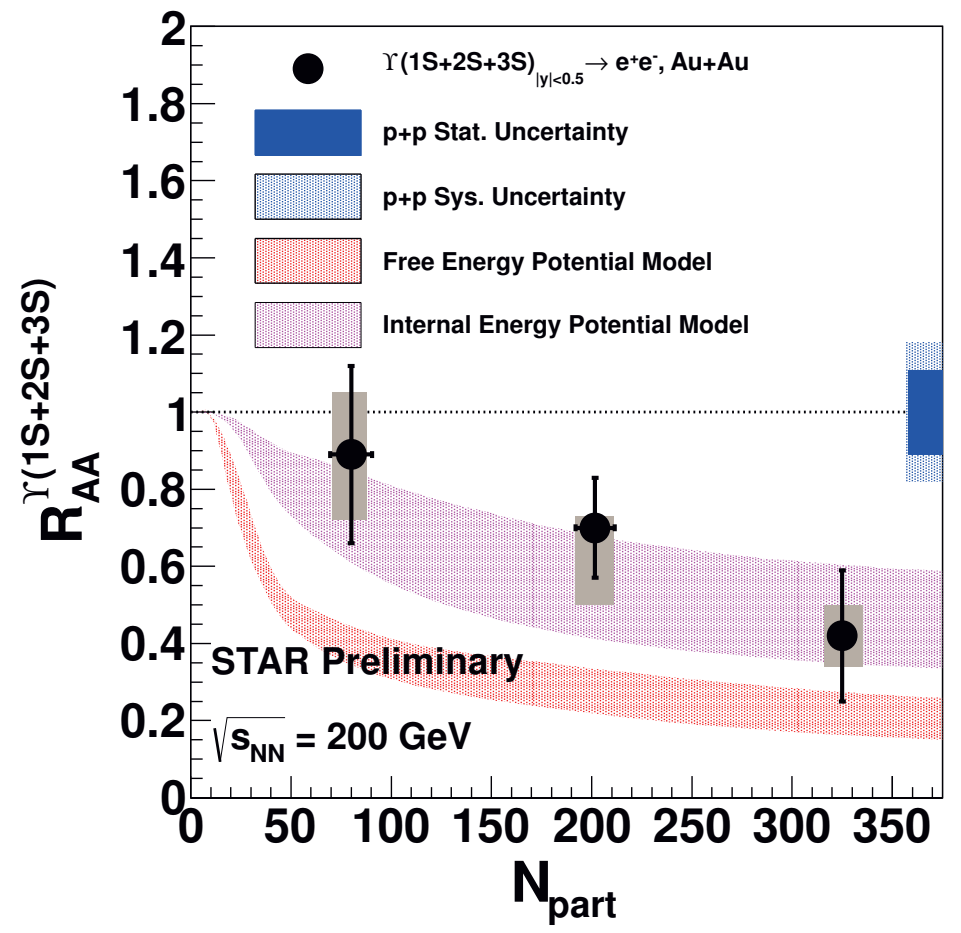

Figure 5. The nuclear modification factor of $\Upsilon(1 \mathrm{~S}, 2 \mathrm{~S}, 3 \mathrm{~S})$ production as a function of the number of participants in $200 \mathrm{GeV} \mathrm{Au+Au}$ collisions measured by the STAR experiment. Taken from Ref. [19].

\section{References}

[1] J. Adams et al. [STAR Collaboration], Phys. Rev. Lett. 94, 062301 (2005).

[2] S. Baumgart et al. [ STAR Collaboration ], Eur. Phys. J. C62, 3 (2009).

[3] B. I. Abelev et al. [STAR Collaboration], arXiv:0805.0364 [nucl-ex].

[4] L. Adamczyk et al. [STAR Collaboration], Phys. Rev. D 86, 072013 (2012).

[5] D. Tlusty et al. [STAR Collaboration], Quark Matter 2012, August 13-18, 2012, Washington, USA.

[6] M. Cacciari, P. Nason and R. Vogt, Phys. Rev. Lett. 95, 122001 (2005).

[7] B. I. Abelev et al. [STAR Collaboration ], Phys. Rev. Lett. 98, 192301 (2007).

[8] A. Adare et al. [PHENIX Collaboration], Phys. Rev. C 84, 044905 (2011).

[9] M. M. Aggarwal et al. [STAR Collaboration ], Phys. Rev. Lett. 105, 202301 (2010).

[10] H. Agakishiev et al. [STAR Collaboration ], Phys. Rev. D83, 052006 (2011).

[11] A. Adare et al. [PHENIX Collaboration], Phys. Rev. Lett. 97, 252002 (2006).

[12] A. Adare et al. [PHENIX Collaboration], arXiv:1208.1293 [nucl-ex].

[13] T. Matsui, H. Satz, Phys. Lett. B178, 416 (1986).

[14] A. Adare et al. [PHENIX Collaboration], Phys. Rev. C 84, 054912 (2011). 
[15] F. Karsch, D. Kharzeev, H. Satz, Phys. Lett. B637, 75 (2006).

[16] A. Adare et al. [PHENIX Collaboration], Phys. Rev. Lett. 107, 142301 (2011).

[17] A. Adare et al. [PHENIX Collaboration], arXiv:1204.0777 [nucl-ex].

[18] B. I. Abelev et al. [STAR Collaboration], Phys. Rev. C 80, 041902 (2009).

[19] B. Trzeciak et al. [STAR Collaboration], Quark Matter 2012, August 13-18, 2012, Washington, USA.

[20] B. I. Abelev et al. [STAR Collaboration], Phys. Rev. D82, 012004 (2010).

[21] H. Liu [STAR Collaboration], Nucl. Phys. A 830, 235C (2009).

[22] R. Reed [STAR Collaboration], Nucl. Phys. A855, 440 (2011). 